\title{
Achieving Low-overhead Fault Tolerance for Parallel Accelerators with Dynamic Partial Reconfiguration
}

\author{
James J. Davis and Peter Y. K. Cheung \\ Department of Electrical and Electronic Engineering \\ Imperial College London \\ London, SW7 2AZ, United Kingdom \\ E-mail: \{james.davis06, p.cheung\}@imperial.ac.uk
}

\begin{abstract}
While allowing for the fabrication of increasingly complex and efficient circuitry, transistor shrinkage and countper-device expansion have major downsides: chiefly increased variation, degradation and fault susceptibility. For this reason, design-time consideration of fault tolerance will have to be given to increasing numbers of electronic systems in the future to ensure yields, reliabilities and lifetimes remain acceptably high. Many commonly implemented operators are suited to modification resulting in datapath error detection capabilities with far lower area requirements. FPGAs are uniquely placed to allow further area savings to be made when incorporating fault avoidance mechanisms thanks to their dynamic reconfigurability.
\end{abstract}

In this paper, we examine the practicalities and costs involved in implementing hardware-software fault tolerance on a test platform: a parallel matrix multiplication accelerator in hardware, with controller in software, running on a Xilinx Zynq system-on-chip. A combination of 'bolt-on' error detection logic and software-triggered routing reconfiguration serve to provide low-overhead datapath fault tolerance at runtime. Rapid yet accurate fault diagnoses along with low hardware (area), software (configuration storage) and performance penalties are achieved.

\section{INTRODUCTION}

While the structure of FPGAs make them highly suitable for the realisation of high-performance hardware, typically through parallelisation and pipelining, their high transistor counts and reliance upon RAM for configuration storage make them particularly sensitive to the effects of variation and degradation. Their runtime reconfigurability, however, allows the application of unique strategies for fault correction. Here, we demonstrate the ability to reduce algorithmic parallelisation at runtime in order to maintain accurate operation, using both algorithm-based fault tolerance (ABFT) and dynamic partial reconfiguration (DPR) techniques to do so while keeping the overheads incurred as a result low.

The key contributions of this work are

- the first implementation of ABFT-protected hardware using DPR for recovery in the presence of failure, and

- a quantitative analysis of the overheads - of resources, memory and performance-incurred through the incorporation of that fault tolerance strategy into a benchmark hardware accelerator.

\section{BACKGROUND}

\section{A. Runtime Fault-tolerant Methods}

A wide array of fault detection and correction techniques exist that are specific both to FPGAs and to ASICs in general. A comprehensive analysis of fault-tolerant methods specific to FPGAs was published recently [1], and those of particular relevance to this research are reviewed here. Table I places this work within a side-by-side comparison of competing families of fault tolerance strategies.

Modular redundancy, particularly triple (TMR), remains popular. While design vulnerability to faults is low for those protected by TMR, its application comes with a high costover $200 \%$ area overhead-and its ability to mask faults breaks down when they occur in more than one replicated module simultaneously. Fault detection is possible with singly replicated modules, as in duplicate-with-compare (DWC), but correction is not. Concurrent error detection (CED) schemes generally have lower overheads but suffer from confounding: often, many faults produce the same error codes.

The reconfigurability of FPGAs presents many interesting options for runtime fault tolerance. DPR can be used to allow portions of an FPGA to be tested while the remainder continues to function as normal [2]. While high (up to $96 \%$ for logic [3] and 99\% for interconnect [4]) fault coverage has been achieved, such 'roving' schemes introduce limitations and complications to the operating configuration: the available resources are reduced in number, path-lengthening forces frequency penalties to be incurred $(2.5-15.1 \%$ decreases in frequency have been reported [2]) and care must be taken to avoid glitches when resource substitution takes place. Detection latencies are limited by the rate of chip scanning.

Given the impracticalities of performing on-chip placeand-route, repair strategies tend to involve the storage of precompiled alternative configurations [5] or design-time reservation of spare resources [6] that allow the reorganisation of logic to avoid faults while maintaining identical operation. Where recompilation at runtime is required, time and power requirements can be reduced by containing repairs within subsections of an FPGA [7]. Many faults cannot be tolerated, however, and spare resource overheads are often high.

\section{B. Algorithm-based Fault Tolerance}

The application of fault tolerance at a level above transistors, gates or small circuits-that is, at an algorithmic level-makes it possible to reduce the impacts, in terms of resource usage, performance or both, of those mechanisms while ensuring that reliability remains high. Many linear algebra operations, common in FPGA applications, can be protected with such algorithm-based techniques: examples include matrix operations [8] and Fourier transformations [9]. Recently published work [10] focussed on design vulnerability reduction using $\mathrm{ABFT}$ applied to a matrix multiplier on an FPGA. Our previous work [11], meanwhile, used ABFT for error detection in the same operator while adding additional, fixed logic for dynamic resource reallocation to facilitate fault 
TABLE I. COMPARISON OF RUNTIME FAULT-TOLERANT METHODS

\begin{tabular}{|c|c|c|c|c|c|c|}
\hline \multirow{2}{*}{ Method } & \multirow{2}{*}{ Detection } & \multirow{2}{*}{ Correction } & \multirow{2}{*}{$\begin{array}{l}\text { Fault } \\
\text { types }\end{array}$} & \multicolumn{2}{|c|}{ Overheads } & \multirow{2}{*}{ Limitations } \\
\hline & & & & Area & Performance & \\
\hline Scrubbing & - & Reconfiguration & Transient & - & - & (Must be paired with detection scheme) \\
\hline DWC & Redundancy & - & All & High $(>100 \%)$ & Low & No fault locatability \\
\hline TMR & Redundancy & Masking & All & High $(>200 \%)$ & Low & Becomes DWC after single failure \\
\hline CED & Parity (or similar) & - & All & Low & Low & Low fault detectability \\
\hline Re-execution & Time redundancy & Re-execution & Transient & Low-moderate & High $(>100 \%)$ & Low-moderate detection latency \\
\hline Roving & Test vectors & (Reconfiguration) & All & Low-moderate & Moderate & Forces path-lengthening, low detection latency \\
\hline ABFT & Algorithmic & (Algorithmic) & All & Low-moderate & Moderate & Algorithm-specific \\
\hline This work & Algorithmic & Reconfiguration & All & Low-moderate $(\approx 10 \%)$ & Moderate $(\approx 25 \%)$ & Algorithm-specific \\
\hline
\end{tabular}

avoidance at runtime. In this work, we call upon ABFT once again for its low-overhead error detection properties but make use of DPR for the purposes of fault avoidance.

Matrix multiplication was chosen as a case study in this work since it is used in many hardware-accelerated applications and because the adaptation of its operation for ABFT is straightforward. In order to protect the operation $Q=A B$, two new matrices- $A^{\prime}$ and $B^{\prime}$-are formed such that

$A^{\prime}=\left(\begin{array}{ccc}a_{1,1} & \cdots & a_{1, N} \\ \vdots & \ddots & \vdots \\ a_{N, 1} & \cdots & a_{N, N} \\ \sum_{n=1}^{N} a_{n, 1} & \cdots & \sum_{n=1}^{N} a_{n, N}\end{array}\right), B^{\prime}=\left(\begin{array}{cccc}b_{1,1} & \cdots & b_{1, N} & \sum_{n=1}^{N} b_{1, n} \\ \vdots & \ddots & \vdots & \vdots \\ b_{N, 1} & \cdots & b_{N, N} & \sum_{n=1}^{N} b_{N, n}\end{array}\right)$

$A^{\prime}$ is an expanded version of $A$ : its $(N+1)^{t h}$ row comprises checksums of its previous rows' values on a columnby-column basis. Conversely, the $(N+1)^{\text {th }}$ column of $B^{\prime}$ comprises checksums of its previous columns' values on a row-by-row basis. $A^{\prime} B^{\prime}$ yields

$Q^{\prime}=\left(\begin{array}{cccc}q_{1,1} & \cdots & q_{1, N} & \sum_{n=1}^{N} q_{1, n} \\ \vdots & \ddots & \vdots & \vdots \\ q_{N, 1} & \cdots & q_{N, N} & \sum_{n=1}^{N} q_{N, n} \\ \sum_{n=1}^{N} q_{n, 1} & \cdots & \sum_{n=1}^{N} q_{n, N} & \sum_{n=1}^{N} q_{n, N+1}^{\prime}=\sum_{n=1}^{N} q_{N+1, n}^{\prime}\end{array}\right)$

The $(N+1)^{t h}$ row and column of $Q^{\prime}$ comprise column and row checksums, respectively, of the same format as those in $A^{\prime}$ and $B^{\prime}$. Post-multiplication verification of the checksums contained within $Q^{\prime}$ allows the result obtained to be confirmed as accurate to within a high degree of confidence. Consider

$$
Q=A B=\left(\begin{array}{ll}
1 & 2 \\
3 & 4
\end{array}\right) \times\left(\begin{array}{ll}
5 & 6 \\
7 & 8
\end{array}\right)=\left(\begin{array}{ll}
19 & 22 \\
43 & 50
\end{array}\right)
$$

which, once input checksumming has been added, becomes

$Q^{\prime}=A^{\prime} B^{\prime}=\left(\begin{array}{ll}1 & 2 \\ 3 & 4 \\ \mathbf{4} & \mathbf{6}\end{array}\right) \times\left(\begin{array}{ccc}5 & 6 & \mathbf{1 1} \\ 7 & 8 & \mathbf{1 5}\end{array}\right)=\left(\begin{array}{ccc}19 & 22 & \mathbf{4 1} \\ 43 & 50 & \mathbf{9 3} \\ \mathbf{6 2} & \mathbf{7 2} & \mathbf{1 3 4}\end{array}\right)$

Throughout this paper, $A, B$ and $Q$ will have dimensions $N \times N$, however this is merely for simplification and not a requirement imposed upon the hardware by this method.

\section{IMPLEMENTATION}

\section{A. System Overview}

The fault-tolerant system developed, a high-level overview of which is presented in Figure 1, was implemented wholly upon a Xilinx Zynq-7000 XC7Z020 system-on-chip (SoC). Our hardened matrix multiplication accelerator sits on the programmable logic (PL) portion of the device. A region of the accelerator-represented by a dashed rectangle-is reconfigurable. Wrapped around the accelerator are block RAM (BRAM) and direct memory access (DMA) controllers to facilitate data transfers between the accelerator and off-chip dynamic RAM (DRAM). On the SoC's processor subsystem (PS) side, a single ARM core is used to trigger memory transfers, accelerator runs and necessary reconfigurations via a software driver. The processor is also used for execution time measurement and result verification. While used here, an embedded processor is not a requirement: a soft core or fully customised logic implemented upon any dynamically reconfigurable FPGA could be used in its place, although each would require an increase in design effort.

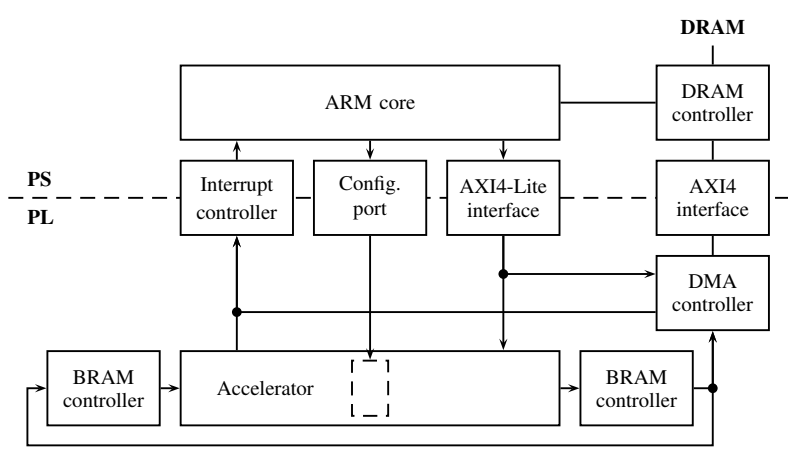

Fig. 1. System block diagram

\section{B. Matrix Multiplication Datapath}

The accelerator's datapath is shown in Figure 2. At the its heart are $N+1$ parallel multiply-accumulators (MACs) one per column of $Q^{\prime}$-allowing matrix multiplication to be reduced from an $O\left(N^{3}\right)$ operation to $O\left(N^{2}\right)$. Wide $(D N$ - and $D(N+1)$-bit, for $D$-bit matrix element data) BRAMs are used to allow complete matrix row access on a cycle-by-cycle basis. Checksum generation and verification logic, explained in Section III-C, and reconfigurable routing, represented by dashed squares and explained in Section III-E, are added to harden the datapath.

While data width $D$, matrix dimensionality $N$, multiplier and RAM styles and multiplier and accumulator pipeline depths are customisable, for this work only $N$ was varied. 32bit fixed-point data was used here, along with DSP multipliers and BRAMs for memories. 15-stage multipliers and singlestage accumulators were experimentally revealed to be ideal.

\section{Error Detection}

Before a multiplication begins, rows of $B$ are fetched in turn in order to precompute their checksums. This is 


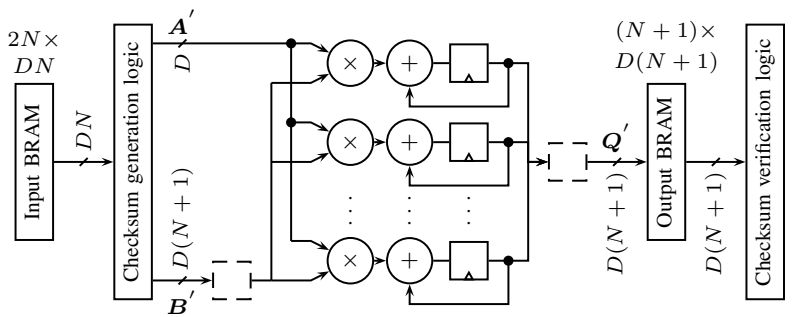

Fig. 2. Fault-tolerant datapath

accomplished by the adder, $D$-bit register and row checksum RAM shown in Figure 3. Once complete, the first row of $A$ is buffered into the $D N$-bit register, following which rows of $B^{\prime}$ are fed to the MACs for computation. $A$ checksum generation - accomplished by the adder and column checksum RAM-occurs on-the-fly as its columns are accessed. This process repeats until all rows of $A$ have been consumed. Once complete rows of $Q^{\prime}$ have been written into the output BRAM, they are fetched in turn in order to be verified in a similar manner by the logic shown in Figure 4.

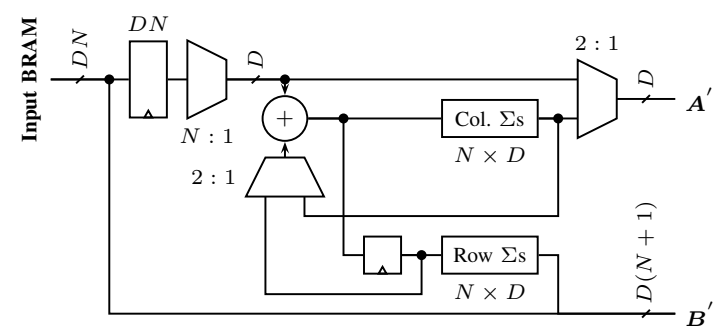

Fig. 3. Checksum generation logic

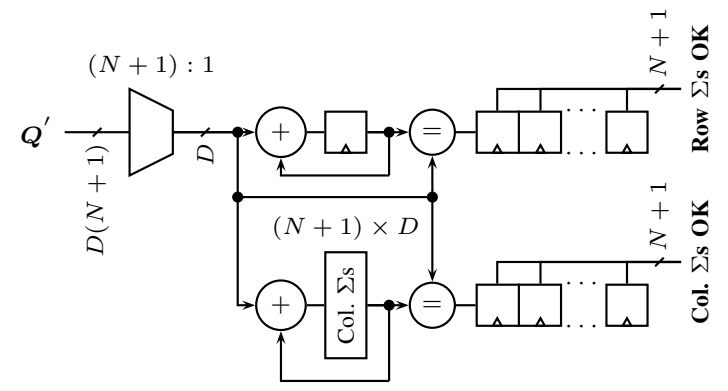

Fig. 4. Checksum verification logic

\section{Fault Location}

Had the multiplication in Section II-B resulted in

$Q^{\prime}=\left(\begin{array}{ccc}\mathbf{2 1} & 22 & 41 \\ \mathbf{4 5} & 50 & 93 \\ \mathbf{6 3} & 72 & 134\end{array}\right),\left(\begin{array}{ccc}19 & \mathbf{2 3} & 41 \\ 43 & \mathbf{5 1} & 93 \\ 62 & \mathbf{7 3} & 134\end{array}\right)$ or $\left(\begin{array}{ccc}19 & 22 & \mathbf{4 3} \\ 43 & 50 & \mathbf{9 5} \\ 62 & 72 & \mathbf{1 3 5}\end{array}\right)$

instead, the positioning of incorrect checksum values would have revealed location information regarding the MACs that caused the errors. Each of these three cases is synonymous with a single MAC register's least-significant bit experiencing a stuck-at-one fault. Elements that have been calculated incorrectly are shown in bold, while italics mark error-indicating checksum values. Note that column checksum mismatches relate one-to-one with faulty MACs, since each MAC is responsible for computing exactly one output column's elements. Simultaneous faults occurring both within an individual MAC and across multiple MACs would yield equally informative results: a single column checksum mismatch in the former case and multiple in the latter.

\section{E. Fault Avoidance}

While our previously published matrix multiplication accelerator made use of additional logic to dynamically reallocate data to the MACs in order to avoid faults, here we achieve the same end result with partial routing reconfiguration. During accelerator runs in which at least one error is detected, fault location data is sent back to the controlling driver in order to facilitate corrective action. Based upon the locations of faults observed and, conversely, the locations of remaining healthy MACs, one or more rounds of routing reconfiguration followed by multiplier reruns, together called 'corrective runs,' can be performed in order to re-establish accurate operation.

At compile-time, routing configurations representing different amounts of data-shifting are synthesised along with the rest of the design, which remains static. Nets $B^{\prime}$ and $Q^{\prime}-$ shown in Figure 2 - are broken and routed via a single reconfigurable partition, which then dictates the data connections on both the input and output sides of the accelerator's datapath. Figure 5 shows the configurations available for the multiplier when $N=2$. Circled numbers represent MACs, while the input and output halves of the reconfigurable partition are shown as dashed rectangles. In each case, the output shifting arrangement mirrors that on the input side.

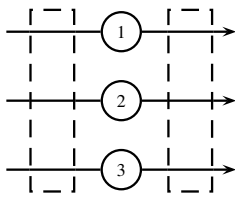

0-place shift

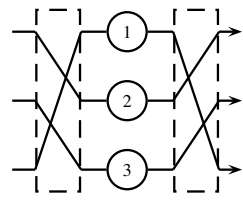

1-place shift

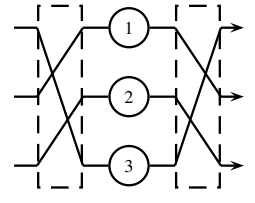

2-place shift
Fig. 5. Routing configurations available when $N=2$

When routing reconfiguration is required, the driver initiates a partial bitstream transfer, via the processor configuration access port, from DRAM to the FPGA fabric. In order to lower the total number of configurations required, only configurations with equal-place shifting per MAC are generated. The number of configurations stored for each accelerator is therefore $N+1$.

Our driver currently supports two levels of safety for error correction. When operating in the safer mode, all incorrectly computed columns of $Q^{\prime}$ are recalculated, after which checksum verification is repeated to confirm successful correction. In the less safe mode, the ABFT mechanism is essentially turned off: the $(N+1)^{t h}$ MAC becomes a usable spare and the output is assumed to be accurate once corrective runs complete. As a consequence of this, faults that affect only the $(N+1)^{t h}$ MAC are ignored in the less safe mode. The choice made between these modes in a real application would be based upon the likelihood of additional faults developing in different MACs during the time it takes to complete a corrective run. Note that re-transfer of input data and input checksum regeneration are not required in either mode.

Figure 6 demonstrates the application of routing reconfiguration in order to avoid a single faulty MAC - labelled 2when $N=2$. Intuitively, one corrective run is required to 
overwrite the second column's elements. A single-place shift allows MAC 3 to perform the recalculation required.

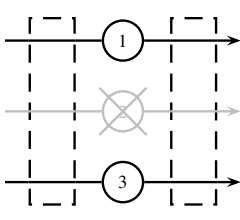

Step 1

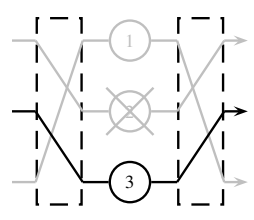

Step 2
Fig. 6. Steps for avoidance of a single faulty MAC when $N=2$

In cases of multiple faults, differing amounts of datashifting are required. This is exemplified in Figure 7, in which six different combinations of double-fault locations are shown for $N=4$. In the three leftmost cases, one single-place shift is required, while in the three rightmost cases, one double-place shift is required. Curved arrows represent the reallocation of resources necessary during a corrective run.

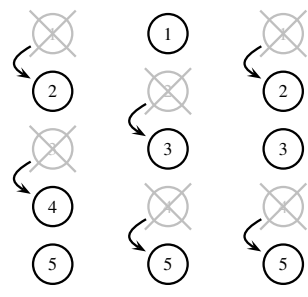

1-place shift required

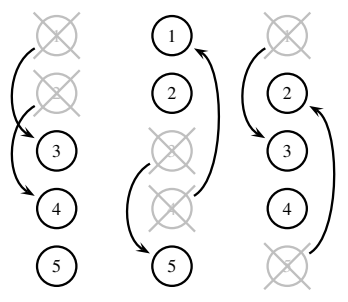

2-place shift required
Fig. 7. Example double-fault locations requiring differing routing reconfigurations when $N=4$

Intuition may suggest that the number of corrective runs required is only dependant upon the ratio of faulty to healthy MACs. When $N \in\{2,4\}$, this is indeed true, but for $N \geq$ 8 the situation is more complicated since there are cases in which a configuration with an equal-place shift per MAC can no longer match all faulty MACs to remaining healthy ones. In Figure 8, where $N=8$, six combinations of quadruplefault locations are shown. In the three leftmost cases, only a single corrective run is required; in the three rightmost cases, however, two are needed: resource reallocations which cannot be performed in the first run are represented by dashed lines.

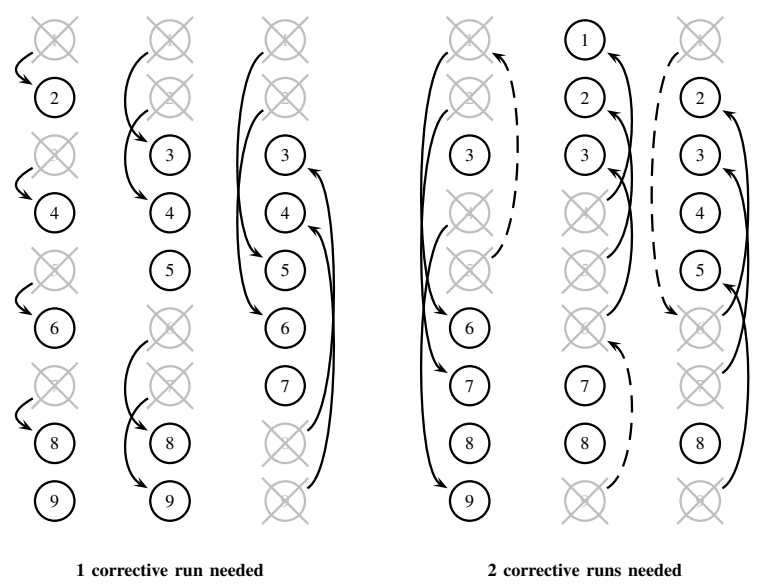

Fig. 8. Example quadruple-fault locations requiring differing numbers of routing reconfigurations when $N=8$

\section{EXPERIMENTS AND RESULTS}

\section{A. Hardware Overheads}

All designs were synthesised with version 14.7 of Xilinx's ISE toolchain. Table II contains the raw resource usage figures obtained for all implementations-including the faultintolerant, fault-tolerant via additional logic from our previous work and fault-tolerant via DPR versions of the acceleratorper resource type. Percentages of the total number of each of these resources for the target device are also included, along with a mean of the individual proportions to give an indication of the overall resource utilisation. The timing model $f_{\max }$ of each design is also given. Figure 9 presents a visual summary of the combined resource usage data.

TABLE II. RAW RESOURCE USAGE AND $f_{\max }$ FOR VARYING $N$

\begin{tabular}{|c|c|c|c|c|c|c|c|}
\hline$N$ & $\begin{array}{c}\text { Fault } \\
\text { avoidance }\end{array}$ & Registers & LUTs & BRAMs & DSPs & $\begin{array}{c}\text { Total } \\
\text { resources }\end{array}$ & $\begin{array}{l}f_{\max } \\
(\mathrm{MHz})\end{array}$ \\
\hline \multirow{3}{*}{2} & None & $\begin{array}{c}210 \\
(0.197 \%)\end{array}$ & $\begin{array}{c}239 \\
(0.449 \%)\end{array}$ & $\begin{array}{c}2 \\
(0.714 \%)\end{array}$ & $\begin{array}{c}6 \\
(2.73 \%)\end{array}$ & $1.02 \%$ & 90.204 \\
\hline & $\operatorname{Logic}^{1}$ & $\begin{array}{c}597 \\
(0.561 \%)\end{array}$ & $\begin{array}{c}665 \\
(1.25 \%)\end{array}$ & $\begin{array}{c}5 \\
(1.79 \%)\end{array}$ & $\begin{array}{c}9 \\
(4.09 \%)\end{array}$ & $1.92 \%$ & 88.992 \\
\hline & $\mathrm{DPR}^{2}$ & $\begin{array}{c}406 \\
(0.382 \%)\end{array}$ & $\begin{array}{c}711 \\
(1.34 \%)\end{array}$ & $\begin{array}{c}5 \\
(1.79 \%)\end{array}$ & $\begin{array}{c}9 \\
(4.09 \%)\end{array}$ & $1.90 \%$ & 95.712 \\
\hline \multirow{3}{*}{4} & None & $\begin{array}{c}406 \\
(0.382 \%)\end{array}$ & $\begin{array}{c}441 \\
(0.829 \%)\end{array}$ & $\begin{array}{c}8 \\
(2.86 \%)\end{array}$ & $\begin{array}{c}12 \\
(5.45 \%)\end{array}$ & $2.38 \%$ & 80.103 \\
\hline & Logic & $\begin{array}{c}945 \\
(0.888 \%)\end{array}$ & $\begin{array}{c}855 \\
(1.61 \%)\end{array}$ & $\begin{array}{c}11 \\
(3.93 \%)\end{array}$ & $\begin{array}{c}15 \\
(6.82 \%)\end{array}$ & $3.31 \%$ & 88.168 \\
\hline & DPR & $\begin{array}{c}620 \\
(0.583 \%)\end{array}$ & $\begin{array}{c}898 \\
(1.69 \%)\end{array}$ & $\begin{array}{c}11 \\
(3.93 \%)\end{array}$ & $\begin{array}{c}15 \\
(6.82 \%)\end{array}$ & $3.25 \%$ & 82.102 \\
\hline \multirow{3}{*}{8} & None & $\begin{array}{c}794 \\
(0.746 \%)\end{array}$ & $\begin{array}{c}604 \\
(1.14 \%)\end{array}$ & $\begin{array}{c}16 \\
(5.71 \%)\end{array}$ & $\begin{array}{c}24 \\
(10.9 \%)\end{array}$ & $4.63 \%$ & 76.941 \\
\hline & Logic & $\begin{array}{c}1625 \\
(1.53 \%) \\
\end{array}$ & $\begin{array}{c}2037 \\
(3.83 \%)\end{array}$ & $\begin{array}{c}19 \\
(6.79 \%)\end{array}$ & $\begin{array}{c}27 \\
(12.3 \%)\end{array}$ & $6.10 \%$ & 77.042 \\
\hline & DPR & $\begin{array}{c}1036 \\
(0.974 \%)\end{array}$ & $\begin{array}{c}1375 \\
(2.58 \%)\end{array}$ & $\begin{array}{c}19 \\
(6.79 \%)\end{array}$ & $\begin{array}{c}27 \\
(12.3 \%)\end{array}$ & $5.65 \%$ & 85.918 \\
\hline \multirow{3}{*}{16} & None & $\begin{array}{c}1566 \\
(1.47 \%)\end{array}$ & $\begin{array}{c}613 \\
(1.15 \%)\end{array}$ & $\begin{array}{c}30 \\
(10.7 \%)\end{array}$ & $\begin{array}{c}48 \\
(21.8 \%)\end{array}$ & $8.79 \%$ & 50.495 \\
\hline & Logic & $\begin{array}{c}2970 \\
(2.79 \%)\end{array}$ & $\begin{array}{c}1674 \\
(3.15 \%)\end{array}$ & $\begin{array}{c}33 \\
(11.8 \%)\end{array}$ & $\begin{array}{c}51 \\
(23.2 \%)\end{array}$ & $10.2 \%$ & 56.850 \\
\hline & DPR & $\begin{array}{c}1874 \\
(1.76 \%) \\
\end{array}$ & $\begin{array}{c}2273 \\
(4.27 \%) \\
\end{array}$ & $\begin{array}{c}33 \\
(11.8 \%) \\
\end{array}$ & $\begin{array}{c}51 \\
(23.2 \%) \\
\end{array}$ & $10.3 \%$ & 52.062 \\
\hline \multirow{3}{*}{32} & None & $\begin{array}{c}3105 \\
(2.92 \%)\end{array}$ & $\begin{array}{c}2115 \\
(3.98 \%)\end{array}$ & $\begin{array}{c}58 \\
(20.7 \%)\end{array}$ & $\begin{array}{c}96 \\
(43.6 \%)\end{array}$ & $17.8 \%$ & 58.156 \\
\hline & Logic & $\begin{array}{c}5643 \\
(5.30 \%)\end{array}$ & $\begin{array}{c}4203 \\
(7.90 \%)\end{array}$ & $\begin{array}{c}61 \\
(21.8 \%)\end{array}$ & $\begin{array}{c}99 \\
(45.0 \%)\end{array}$ & $20.0 \%$ & 55.857 \\
\hline & DPR & $\begin{array}{c}3675 \\
(3.45 \%)\end{array}$ & $\begin{array}{c}4363 \\
(8.20 \%)\end{array}$ & $\begin{array}{c}61 \\
(21.8 \%)\end{array}$ & $\begin{array}{c}99 \\
(45.0 \%)\end{array}$ & $19.6 \%$ & 53.101 \\
\hline
\end{tabular}

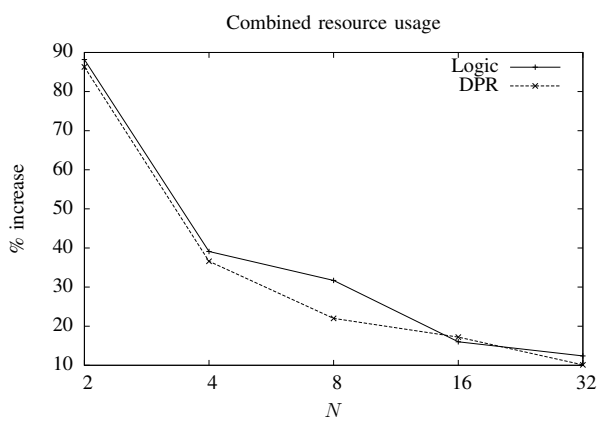

Fig. 9. Combined resource usage overhead for varying $N$ versus faultintolerant hardware

With the exception of $N=16$, it is clear from Figure 9 that the DPR-shifting accelerator betters its logic-shifting counterpart for overall resource usage across the range of $N$ tested. Since BRAM and DSP usage are identical between the two versions, register and LUT counts are accountable for all differences in utilisation. As expected, LUT overhead for the DPR design decreases proportionally as $N$ increases

${ }^{1}$ Previous approach [11]

${ }^{2}$ Proposed approach 
thanks to the elimination of the circular shifters present in the logic-shifting version. Conversely, register overhead tends to increase slightly; this is due to pipelining registers inserted into the lengthened paths that pass through the reconfigurable region, the number of which increases linearly with $N$. For our largest-tested design, $N=32$, the DPR-shifting accelerator achieved an overall area overhead of $10.1 \%-17.7 \%$ lower than its logic-shifting equivalent. Between these two faulttolerant designs, LUT overhead decreased by $77.5 \%$ while register overhead increased by $7.7 \%$. $f_{\max }$ changes are not well correlated, likely due to the stochastic nature of the placement and routing tools used, although decreases between the logic- and DPR-shifting designs due to path-lengthening are seen for larger $N$.

\section{B. Software Overheads}

From a software perspective, the primary overhead of our fault tolerance strategy is partial bitstream storage. Since accelerator data and bitstream transfers, as well as accelerator runs, are interrupt-driven, their hits on CPU performance are negligible. Table III summarises the DRAM storage requirements for each value of $N$ tested. The size of each partial bitstream is given along with the total storage requirement for that value of $N$. The memory occupation is also expressed, for each $N$, as a proportion of the DRAM available (512MB) on the development board used.

TABLE III. BITSTREAM STORAGE REQUIREMENTS FOR VARYING $N$

\begin{tabular}{|c|c|c|}
\hline \multirow{2}{*}{$N$} & \multicolumn{2}{|c|}{ Bitstream size (kB) } \\
\cline { 2 - 3 } & Each & Total \\
\hline 2 & 15.2 & $45.7(0.00871 \%)$ \\
\hline 4 & 29.4 & $147(0.0281 \%)$ \\
\hline 8 & 43.6 & $393(0.0749 \%)$ \\
\hline 16 & 87.1 & $1480(0.282 \%)$ \\
\hline 32 & 158 & $5220(0.995 \%)$ \\
\hline
\end{tabular}

\section{Performance Impacts}

Testing was performed on the hardware in order to measure its impact upon performance under a number of conditions. Table IV summarises the results of all of these performance tests. Each test was completed 10,000 times; the mean of these runs is given in all cases. Prior to each test, uniformly distributed random input data was generated to form $A$ and $B$. Execution times were measured using a cycle-accurate ARM timer peripheral. In all cases, the FPGA fabric was clocked at $50 \mathrm{MHz}$. Included in Table IV are execution times for the fault-intolerant multiplier, the fault-tolerant via data-shifting accelerator and our DPR-enabled version running in both of its operating modes. Where appropriate, latency increases relative to the equivalently sized fault-intolerant design are given for comparison. Execution times are given for the occurrences of singular and double MAC failures - the former for both the data-shifting and DPR hardware, and the latter for the DPR version only. Permanent faults were emulated through the targetted inversion of a single accumulator output bit within either one or two MACs per run, with fault locations also randomly chosen. Plots of the latency increases over the faultintolerant hardware under fault-free, singly and doubly faulty conditions are given in Figure 10.

The results show that, for all $N>4$, the DPR-shifting accelerator outperforms the logic-shifting version under normal, fault-free operation as well as that in the presence of a single failure. The lower penalties seen during fault-free operation are due to the moving of the checksum verification
TABLE IV. RAW PERFORMANCE FIGURES FOR VARYING $N$

\begin{tabular}{|c|c|c|c|c|}
\hline \multirow{2}{*}{$N$} & \multirow{2}{*}{$\begin{array}{c}\text { Fault } \\
\text { avoidance }\end{array}$} & \multicolumn{3}{|c|}{ Execution time $(\mu \mathrm{s})$} \\
\hline & & Fault-free & Single failure & Double failure \\
\hline \multirow{4}{*}{2} & None & 254 & - & - \\
\hline & Logic & $272(+7.09 \%)$ & $300(+18.1 \%)$ & 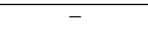 \\
\hline & DPR (less safe) & \multirow{2}{*}{$280(+10.2 \%)$} & $366(+44.1 \%)$ & $451(+77.6 \%)$ \\
\hline & DPR (more safe) & & $392(+54.3 \%)$ & $477(+87.8 \%)$ \\
\hline \multirow{4}{*}{4} & None & 314 & - & - \\
\hline & Logic & $339(+7.96 \%)$ & $398(+26.8 \%)$ & - \\
\hline & DPR (less safe) & \multirow{2}{*}{$351(+11.8 \%)$} & $448(+42.7 \%)$ & $544(+73.2 \%)$ \\
\hline & DPR (more safe) & & $486(+54.8 \%)$ & $581(+85.0 \%)$ \\
\hline \multirow{4}{*}{8} & None & 348 & - & 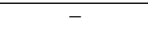 \\
\hline & Logic & $546(+56.9 \%)$ & $712(+105 \%)$ & - \\
\hline & DPR (less safe) & \multirow{2}{*}{$422(+21.3 \%)$} & $557(+60.1 \%)$ & $690(+98.3 \%)$ \\
\hline & DPR (more safe) & & $631(+81.3 \%)$ & $764(+120 \%)$ \\
\hline \multirow{4}{*}{16} & None & 497 & - & - \\
\hline & Logic & $1350(+172 \%)$ & $1910(+284 \%)$ & - \\
\hline & DPR (less safe) & \multirow{2}{*}{$710(+42.9 \%)$} & $982(+97.6 \%)$ & $1254(+152 \%)$ \\
\hline & DPR (more safe) & & $1195(+140 \%)$ & $1467(+195 \%)$ \\
\hline \multirow{4}{*}{32} & None & 3100 & - & - \\
\hline & Logic & $4510(+45.5 \%)$ & $6600(+113 \%)$ & - \\
\hline & DPR (less safe) & \multirow{2}{*}{$3860(+24.5 \%)$} & $4680(+51.0 \%)$ & $5490(+77.1 \%)$ \\
\hline & DPR (more safe) & & $5440(+75.5 \%)$ & $6260(+102 \%)$ \\
\hline
\end{tabular}

logic from the input to the output side of the output BRAM, allowing return data transfers to start (and end) sooner than they had previously, while gains under single failure mode are realised for larger $N$ as reconfiguration times proportionately fall. The relationship between the performance plots for the DPR-shifting version working in its two modes demonstrates the near-fixed performance cost paid by operating more safely. The trend-reversal seen on all plots after $N=16$ can be attributed to data transfer throttling: once $N$ passes 16 , memory copies begin to dominate accelerator execution for proportional runtime. Performance impacts arising from the use of partial reconfiguration are negligible due to the bitstreams' small size and infrequent application per accelerator run. For our largesttested design, $N=32$, the DPR-shifting accelerator incurred a $24.5 \%$ latency penalty under fault-free operation- $46.1 \%$ lower than its logic-shifting equivalent.

\section{Fault Observability}

In order to assess the fault observability of our chosen detection method, fault injection simulations were performed to ascertain the hardware's ability to correctly detect and locate the faults. Detectable faults are those that result in one or more checksum mismatches - in one or more rows, columns or both-while those that are locatable cause mismatches within the columns corresponding to the MACs they have affected.

For each combination of $N$ and data width $\epsilon$ $\{2,4,8,16,32\}$, the following test steps were completed 400,000 times for both single and double faulty MAC emulation. The results of this testing are presented in Figure 11.

1) Uniformly distributed random input data was generated for each element of $A$ and $B$, with checksums calculated to form $A^{\prime}$ and $B^{\prime}$.

2) During each accumulation step of the subsequent multiplication, a single bit in either one (for single fault injection) or two (for double) columns-also randomly selected - was held high to emulate either one or two stuck-at one MAC register output bits.

3) Once complete, output checksums were verified: where all were found to be correct, the fault was recorded as undetected; where either all (for single faults) or all but one (for double) column checksums were found to be correct, it was unlocated.

For single fault injection, in all cases except for $N=2$, data width $=2$, the proportion of undetected faults dropped 

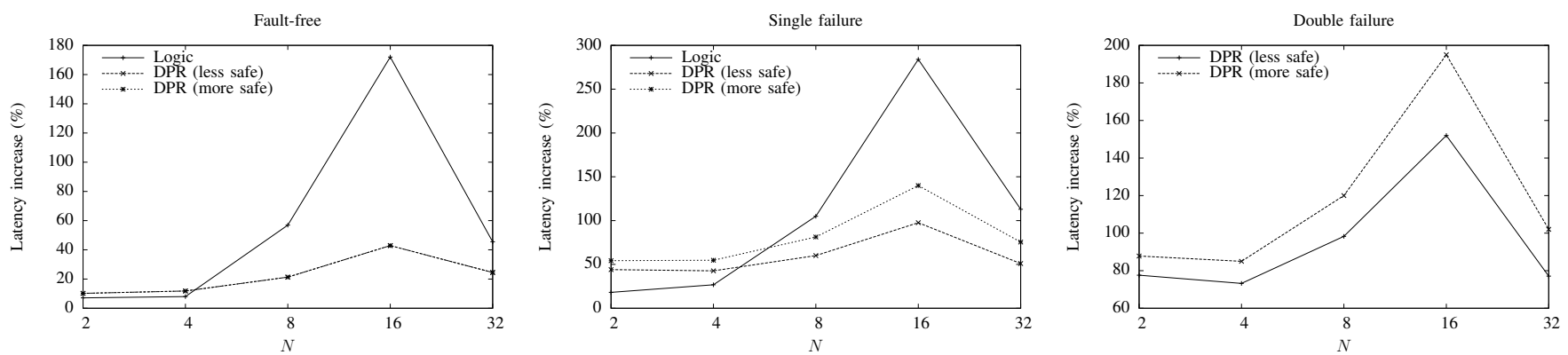

Fig. 10. Performance impact for varying $N$ versus fault-intolerant hardware
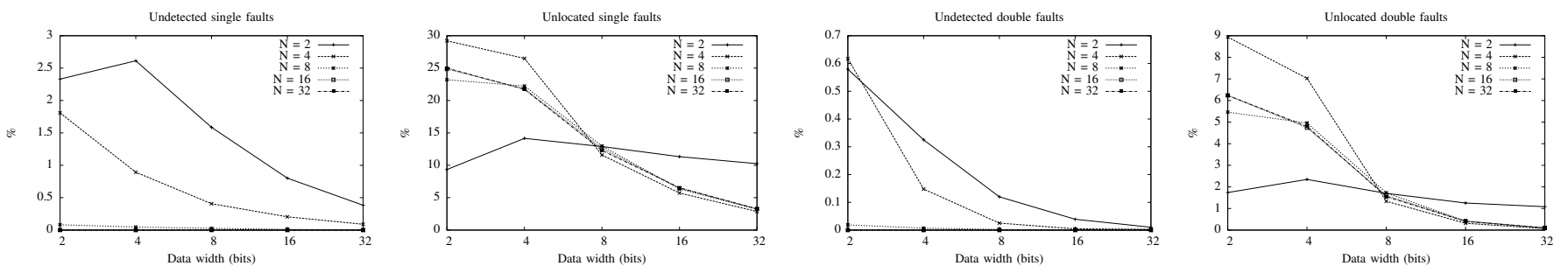

Fig. 11. Fault observability for varying $N$, data width

off with both $N$ and data width. For $N \geq 16$, undetectable fault proportions fell below $0.1 \%$ for all data widths and, for $N=32$, undetectable faults ceased to be encountered. As expected, proportions of unlocatable faults were higher than those that were undetectable due to the lack of redundancy in checksums used for location. In all cases except for $N=2$, data width $=2$, however, the proportion of unlocated faults observed dropped with increasing data width for each value of $N$. For larger $N$, the locatability of faults is largely independent of $N$ itself.

Largely similar trends were seen for double fault injection testing. The rates of both undetected and unlocated faults were all lower, however, for each combination of $N$ and data width. This is expected of undetected faults since the likelihood of errors being masked in multiple columns simultaneously decreases as the number of affected columns increases. The proportions of unlocatable double faults encountered were again significantly higher than those which were undetectable but, for all cases except for $N=2$, data width $=2$, dropped off with increasing data width for all $N$.

\section{CONCLUSion}

In this paper, we explored the combined application of algorithm-based fault tolerance and dynamic partial reconfiguration to a benchmark hardware accelerator-a parallel matrix multiplier-with the goal of achieving a robust, low-overhead design capable of detecting faults within itself and taking corrective action in order to re-establish accurate operation using runtime routing reconfiguration. Our largest implemented ABFT- and DPR-protected design, for the multiplication of two $32 \times 32$ matrices, was found to consume $10.1 \%$ more area and incur a $24.5 \%$ execution time penalty over its equivalent, unprotected design during fault-free operation.

Our future work will focus upon refinements to the accelerator design-including facilitating more comprehensive pipelining and the application of error detection at lower levels of precision-as well as the the generalisation of techniques developed here to allow the protection of other operators. Efforts will also be focussed upon methods of finer-grained fault detection, likely to be performed during periods of temporary downtime on known-faulty functional units, as well as the exploration of the potential application of remote compilation for fault correction.

\section{REFERENCES}

[1] E. Stott, P. Sedcole, and P. Y. K. Cheung, "Fault tolerance and reliability in field-programmable gate arrays," IET Computers and Digital Techniques, vol. 4, no. 3, 2010.

[2] M. Abramovici, C. Strond, C. Hamilton, S. Wijesuriya, and V. Verma, "Using roving STARs for on-line testing and diagnosis of FPGAs in fault-tolerant applications," in International Test Conference, 1999.

[3] S. Dutt, V. Verma, and V. Suthar, "Built-in-self-test of FPGAs with provable diagnosabilities and high diagnostic coverage with application to online testing," IEEE Transactions on Computer-Aided Design of Integrated Circuits and Systems, vol. 27, no. 2, 2008.

[4] V. Suthar and S. Dutt, "Efficient on-line interconnect testing in FPGAs with provable detectability for multiple faults," in Design, Automation and Test in Europe (DATE), vol. 1, 2006.

[5] J. Lach, W. H. Mangione-Smith, and M. Potkonjak, "Low overhead fault-tolerant FPGA systems," IEEE Transactions on Very Large Scale Integration (VLSI) Systems, vol. 6, no. 2, 1998.

[6] F. Hanchek and S. Dutt, "Node-covering based defect and fault tolerance methods for increased yield in FPGAs," in International Conference on VLSI Design, 1996.

[7] V. Lakamraju and R. Tessier, "Tolerating operational faults in clusterbased FPGAs," in International Symposium on Field Programmable Gate Arrays (FPGA), 2000.

[8] K.-H. Huang and J. A. Abraham, "Algorithm-based fault tolerance for matrix operations," IEEE Transactions on Computers, vol. C-33, no. 6, 1984.

[9] S.-J. Wang and N. K. Jha, "Algorithm-based fault tolerance for FFT networks," IEEE Transactions on Computers, vol. 43, no. 7, 1994.

[10] A. Jacobs, G. Cieslewski, and A. D. George, "Overhead and reliability analysis of algorithm-based fault tolerance in FPGA systems," in International Conference on Field Programmable Logic and Applications (FPL), 2012.

[11] J. J. Davis and P. Y. K. Cheung, "Datapath fault tolerance for parallel accelerators," in International Conference on Field-Programmable Technology (FPT), 2013. 